\title{
Pharmacogenetics of thiopurines for inflammatory bowel disease in East Asia: prospects for clinical application of NUDT15 genotyping
}

\author{
Yoichi Kakuta $^{1} \cdot$ Yoshitaka Kinouchi $^{2} \cdot$ Tooru Shimosegawa $^{1}$
}

Received: 19 November 2017/ Accepted: 19 November 2017/Published online: 30 November 2017

(c) The Author(s) 2017. This article is an open access publication

\begin{abstract}
The thiopurine drugs 6-mercaptopurine (6-MP) and azathiopurine (AZA) are widely used to treat inflammatory bowel disease. However, the incidence of adverse reactions is high, particularly in Asia, and the mechanisms of toxicity in Asian populations remain unclear. Thiopurine $S$-methyltransferase (TPMT) is a well-known enzyme that inactivates AZA or 6-MP through methylation and is one of the few pharmacogenetic predictors used in clinical settings in Western countries. Individuals carrying TPMTdeficient genetic variants require reduced drug doses, but this treatment modification is are not applicable to East Asian populations. Several genes code thiopurine-metabolizing enzymes, including TPMT, multidrug-resistance protein 4 , and inosine triphosphatase. These genes have been studied as candidate pharmacogenetic markers; however, it remains unclear why Asian populations seem to be more intolerant than other ethnic groups to a full dose of thiopurines. A genome-wide association approach to identify Asian-specific pharmacogenetic markers in Korean patients with Crohn's disease revealed that a non-synonymous single nucelotide polymorphism in nucleoside diphosphate-linked moiety $X$-type motif 15 (NUDT15) which causes p.Arg139Cys was strongly associated with thiopurine-induced early leukopenia. Six common haplotypes of NUDT15 were reported, and five variants showed medium-to-low enzyme activities, compared with the wild
\end{abstract}

Yoichi Kakuta

ykakuta@med.tohoku.ac.jp

1 Division of Gastroenterology, Tohoku University Graduate School of Medicine, 1-1 Seiryo, Aoba, Sendai 980-8574, Japan

2 Institute for Excellent in Higher Education, Tohoku University, Sendai, Japan haplotype. NUDT15 hydrolyzes the thiopurine active metabolites 6-thio-GTP and 6-thio-dGTP; variants of NUDT15 had lower enzyme activities, causing higher levels of thiopurine active metabolites, resulting in thiopurine-induced leukopenia. In clinical application, NUDT15 genotyping is a good candidate for predicting thiopurine toxicity in East Asian populations. However, the association of NUDT15 diplotypes with thiopurine toxicity remains unclear. Further analyses with large cohorts to confirm the clinical effects of each haplotype are planned.

Keywords Azathiopurine - 6-Mercaptopurine · NUDT15 · Pharmacogenetics $\cdot$ Inflammatory bowel disease

\section{Introduction}

Inflammatory bowel diseases (IBDs), represented by ulcerative colitis and Crohn's disease, are chronic inflammatory intestinal conditions of unknown etiology. The thiopurine drug, 6-mercaptopurine (6-MP), and its prodrug, azathiopurine (AZA), are widely used to treat IBD, and thiopurines are well established as key drugs for sparing steroid therapy and maintaining remission of IBD [1-6]. Additionally, the combined treatment of anti-tumor necrosis factor (TNF) agents with thiopurines has been shown to reduce the risk of anti-drug antibody formation that may diminish response to anti-TNF agents [7-9].

Despite the efficacy of thiopurines, the incidence of adverse reactions is high, particularly in East Asian populations, including Koreans [10], Chinese [11], and Japanese [3, 12]. There are definite racial differences in adverse reaction profiles [13, 14]. For example, the incidence of leukopenia is higher in Asian populations than in Caucasian populations, and hair loss is not uncommon in 
Japanese patients but very rare in Caucasians, although the standard dose of thiopurines in Japan (AZA 1-2 mg/ $\mathrm{kg} / \mathrm{day}$ ) is less than one-half of that used in Europe (AZA $2-2.5 \mathrm{mg} / \mathrm{kg} /$ day) [15]. Some of these adverse reactions are known to be caused by individual differences in thiopurine metabolism, which is affected by the genetic polymorphism of the enzymes [16, 17]. Several pharmacogenetic studies, not only for IBD but also for leukemia and organ transplantation, have been reported [18, 19], and a few of the pharmacogenetic predictors thus identified have been applied in clinical settings [20-22].

Given this background, in this review we focus on recent advances in pharmacogenetic research of thiopurines and the prospects for clinical application of pharmacogenetic markers, paying attention to racial differences.

\section{Thiopurine metabolic pathway}

The metabolic pathways of AZA and 6-MP are well described. These substances are metabolized into their active metabolites through a series of steps [23-26], and 6-thioguanine nucleotides (6-TGNs) consist of 6-T(d)GMP, 6-T(d)GDP, and 6-T(d)GTP. 6-T(d)GTPs are incorporated into DNA (6-TdGTP) and RNA (6-TGTP), causing inhibition of nucleotide and protein synthesis [27, 28] and resulting in immunosuppression. 6-TGTPs also block the Vav-Rac1 pathway in T-cells, inhibiting T-cell-antigen-presenting cell conjugation and the subsequent immune responses [29]. As shown in Fig. 1, there are several thiopurine-metabolizing enzymes, and their activities are partly defined by their genetic polymorphisms. Lower activity of the enzymes that inactivate thiopurines, such as thiopurine $S$-methyltransferase (TPMT), leads to higher production of 6-TGNs or other metabolites [31, 32], causing dose-dependent adverse reactions typified by leukopenia $[16,18,19]$.

\section{Thiopurine $S$-methyltransferase}

Thiopurine $S$-methyltransferase is a well-known enzyme that inactivates AZA or 6-MP by methylation; it is one of the few pharmacogenetic predictors used in clinical settings. TPMT deficiency causes increasing 6-TGN levels related to leukopenia $[16,32]$ and increasing 6-methylmercaptopurine (6-MMP) levels and 6-MMPR levels related to hepatotoxicity [33-36]. There are two ways of testing for TMPT deficiency: enzyme activity or genotype [30]. The first report of inter-individual variations in TPMT enzyme activity identified three levels of activity, namely, high, intermediate, and deficient; in the Caucasian populations tested, approximately $89 \%$ had high enzyme activity and $11 \%$ had intermediate activity, whereas only $0.3 \%$ were deficient [30]. This classification scheme is widely known, but TPMT enzyme activities have since been recognized to be more variable [37]. Genetic polymorphisms cause TPMT deficiency, and more than 40 different variant TPMT alleles $(T P M T * 2-* 41)$ have been reported up to May 2017 [38, 39]. Most of these variants are associated with decreased TPMT activity, relative to the wild allele $\left(T P M T^{*} 1\right)$ [40]. Major TPMT mutant alleles for decreased TPMT activity are TPMT*2, TPMT*3A, and $T P M T * 3 C$ in most populations, with other variants being rare.

The Clinical Pharmacogenetics Implementation Consortium published dosing recommendations for thiopurines based on TPMT genotype [22]. According to these guideline, patients who are heterozygous for the $* 1$ allele and demonstrate reduced activity of the of $* 2 / * 3 A / * 3 C / * 4$ alleles (intermediate methylators) should receive 30-70\% of the full dose (AZA $1-1.5 \mathrm{mg} / \mathrm{kg} /$ day). Those who are homozygous for the alleles showing reduced activity (deficient methylators) should receive $10 \%$ of the full dose at a reduced frequency (every other day administration). TMPT deficiencies that are derived from genetic polymorphisms are robust markers of thiopurine-induced leukopenia. Despite the success of this pharmacogenetic test, a number of major issues remain. One is that the TPMT activity is not defined by a common genotype only [41]. As already mentioned, there are many genotypes of TPMT, and it is difficult to determine a patient's exact genotype using a commercial test. Additionally, there are novel and/or rare variants in coding regions that affect TPMT activity $[39,42,43]$, as well as other genetic variants located in non-coding regions that affect the expression and/or activity levels of the TPMT gene [44, 45]. Furthermore, several co-factors controlling TPMT activities are affected by genetic variations of related genes. Inhibition of the folate cycle affects TPMT activities [46, 47], and genetic variants of methylenetetrahydrofolate reductase (MTHFR), which is associated with folate metabolism, are also associated with TPMT activity [48, 49]. Thus, measuring TPMT activity is a more accurate strategy for predicting the appropriate dose of thiopurines than is TPMT genotyping.

Another issue is ethnicity. The frequencies of these genetic polymorphisms vary in different ethnic groups. A recent extensive whole-genome resequencing of 3554 Japanese individuals found that alleles $* 3 A$ and $* 3 B$ were not present (not observed) and that only the presence of $* 3 C$ could be confirmed $(0.96 \%)$ [50, 51]. Uchiyama et al. reported that TPMT mutant alleles were not detected in 16 intolerant Japanese patients and that TPMT genotypes were not associated with thiopurine-induced leukopenia in a Japanese cohort [52]. Even though there are some very rare 


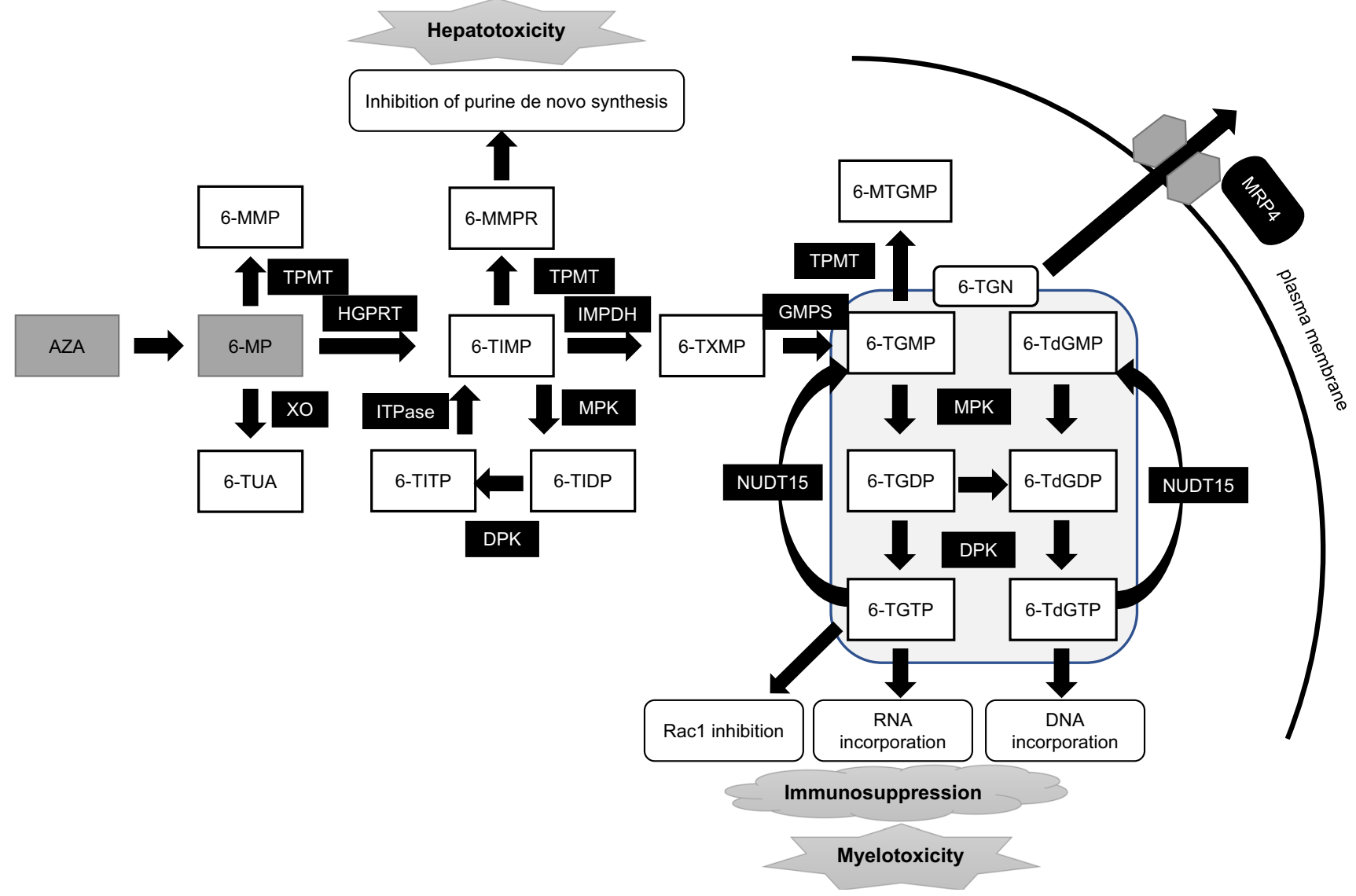

Fig. 1 Thiopurine metabolism and transportation. Drugs are shown in gray boxes: $A Z A$ azathioprine, 6-MP 6-mercaptopurine. Metabolites are shown in white boxes: 6-MMP 6-Methylmercaptopurine, 8-OHMP 8-hydroxy-6-mercaptopurine, 6-TUA 6-thiouric acid, 6-MMPR 6-methylmercaptopurine ribonucleotides, 6-TIMP 6-thioinosine monophosphate, 6-TIDP 6-thioinosine diphosphate, 6-TITP 6-thioinosine triphosphate, 6-TXMP 6-thioxanthosine monophosphate, 6-TGMP 6-thioguanine monophosphate, 6-TGDP 6-thioguanine diphosphate, 6-TGTP 6-thioguanine triphosphate, 6-TdGMP 6-thio-deoxyguanine monophosphate, 6-TdGDP 6-thio-deoxyguanine

variants, such as $* 26$ and $* 30$ in Chinese and Japanese populations [40, 53, 54], TPMT deficiency cannot explain the higher incidence of adverse reactions in East Asian patients.

\section{Multidrug-resistance protein 4 and inosine triphosphatase}

In light of results indicating the absence or low frequency of the TPMT gene in Japanese populations [12, 52, 55], there must be other genetic variations that could explain the frequent thiopurine-induced leukopenia in East Asian populations. Multidrug-resistance protein 4 (MRP4; also known as ABCC4) is a member of a family of multispecific drug transporters and a candidate gene associated diphosphate, 6-TdGTP 6-thio-deoxyguanine triphosphate, 6-MTGMP 6-methylthioguanine monophosphate, 6-TGN 6-thioguaninenucleotides. Enzymes or transporters are shown in black boxes: $X O$ xanthine oxidase, TPMT thiopurine $S$-methyl transferase, HGPRT hypoxanthine phosphoribosyl transferase, IMPDH inosine monophosphate dehydrogenase, GMPS guanosine monophosphate synthetase, $M P K$ monophosphate kinase, DPK diphosphate kinase, ITPase inosine triphosphate pyrophosphatase, MRP4 multidrug resistanceassociated protein 4

with thiopurine metabolism [56]. Mrp4-deficient mice were found to experience thiopurine-induced hematopoietic toxicity caused by the accumulation of 6-TGNs in their myelopoietic cells [57]. MRP is an ATP-dependent efflux pump, therefore Mrp4 may protect thiopurine toxicity by exporting thiopurine metabolites. MRP4-G2269A (rs3765534) causes MRP4 deficiency [57]. 6-TGN levels were found to be significantly higher in patients with MRP4-G2269A, resulting in a significant association with thiopurine-induced leukopenia in Japanese patients with IBDs [58]. However, the MRP4 variant alone could not explain the frequently observed thiopurine toxicity in Asian populations. Interestingly, the MRP4 variant was reported to show gene-gene interactions with inosine triphosphatase (ITPA) and nucleoside diphosphate-linked moiety $X$-type motif 15 (NUDT15) variants [58-60]. 
Variants of the ITPA gene are also candidate predicting markers for adverse events. ITPA is widely expressed in leukocytes and erythrocytes [61, 62], and it catalyzes the hydrolysis of ITPA to prevent the accumulation of 6-thioinosine triphosphate (6-TITP) [61]. 6-TITP would be incorporated into DNA and RNA and compete with nucleotides similar to 6-T(d)GTP of the 6-TGNs. The single nucleotide polymorphisms (SNPs) $94 \mathrm{C}>\mathrm{A}$ and IVS2 + 21A $>$ C, which are associated with ITPA deficiency, have also been found to be associated with thiopurine intolerance, such as leukopenia, flu-like symptoms, and pancreatitis $[63,64]$. These toxicities could be caused by high 6-TITP levels. In Japanese patients with IBD, Uchiyama et al. reported that the ITPA $94 \mathrm{C}>$ A polymorphism was frequent in those patients with thiopurineinduced adverse effects, including leukopenia [52]. Alternatively, ITPA deficiency could reduce the levels of 6-TGNs by reducing the conversion from 6-TITP to 6-thioinosine monophosphate (6-TIMP), resulting in reentry into the 6-MP metabolism pathway. Ban et al. reported that Japanese patients carrying the ITPA 94C > A polymorphism showed significantly lower 6-TGN levels, without leukopenia [58]. Patients carrying both of these MRP4 and ITPA variants did not show leukopenia, and it is possible that the leukopenia associated with MRP4 variants was masked by the opposing effects of ITPA deficiency. The association of ITPA variants with thiopurine toxicity is controversial [65-67], and the impact of ITPA variants does not appear to favor clinical application.

\section{Nucleoside diphosphate-linked moiety $X$-type motif 15}

In addition to TPMT, other genes, including MRP4 and ITPA, have been studied as candidates in Asian populations [10, 12, 52, 55, 68, 69]. However, these genes cannot explain the fact that Asian populations seem to be more intolerant to full doses of thiopurines than Caucasian ones. Therefore, population-specific genetic variants associated with thiopurine intolerance appear to exist. A breakthrough was achieved through a genome-wide association study of Korean patients with Crohn's disease. Yang et al. reported that a non-synonymous SNP in NUDT15 (also known as MTH2) that causes p.Arg139Cys (R139C) was very strongly associated with thiopurine-induced early leukopenia in Koreans [odds ratio (OR) 35.6; $P=4.88 \times 10^{-94}$ ]; these authors also confirmed this association in patients with IBD who were of European ancestry (OR 9.50; $P=4.64 \times 10^{-4}$ ), although it was very rare (minor allele frequency $<0.004$ ) [70]. NUDT15 p.Arg139Cys had a high sensitivity (89.4\%) and specificity (93.2\%) for early leukopenia. The association was also confirmed in populations in Japan, China, India, Thai, Singapore, Guatemala, and Uruguay [71-81]. Furthermore, we have reported that almost all of the patients homozygous for p.Arg139Cys had severe early leukopenia and severe alopecia [72]. Alopecia is known to be a major severe complication in Asian populations, but it is very rare in individuals of European ancestry. Patients who were homozygous for NUDT15 p.Arg139Cys had nearly perfect sensitivity and specificity $(\approx 100 \%)$ for severe alopecia, resembling "Mendelian" drug intolerance [72, 73, 82]. Taking into account the results from previous studies, thiopurines would appear to be contraindicated in patients with IBD who are homozygous for p.Arg139Cys (genotype TT). The incidence of early leukopenia $(<8$ weeks $)$ in patients with IBD who are heterozygous for p.Arg139Cys (genotype CT) was found to be significantly higher than that of patients who are homozygous for the wild-allele (genotype CC) (17.4 vs. 0.93\%); however, there were no significant differences in the continuous rates of thiopurines between genotype $\mathrm{CT}$ and $\mathrm{CC}$ upon dose manipulation [72]. Among Japanese patients with IBD, the average maintenance dose of patients with the $\mathrm{CT}$ genotype was almost one-half of that of the patients with the CC genotype $(0.574 \pm 0.316$ vs. $1.03 \pm 0.425 \mathrm{mg} / \mathrm{kg} /$ day, respectively) [72]. Therefore, testing the genotype of NUDT15 p.Arg139Cys could be useful not only for detecting patients with contraindications for thiopurines (genotype TT), but also to optimize the initial dose of thiopurines.

\section{What does NUDT15 do?}

Most of previously reported genes are associated with thiopurine-induced leukocytopenia with elevated 6-TGN levels. However, Asada et al. reported that there were no differences in 6-TGN levels in patients with each genotype of NUDT15 p.Arg139Cys [73]. Therefore, NUDT15 p.Arg139Cys-related thiopurine-induced leukocytopenia is likely mediated by a 6-TGN-independent mechanism $[73,74,83]$. At the time when the first genome-wide association study was reported, NUDT15 was considered to affect 8-oxo-dGTPase based on the results of only one 2003 study [84]. In 2015, Carter et al. reported that NUDT15 had no effect on the incorporation of 8-oxodGTP into DNA and that it hydrolyzes 6-thio-dGTP, 6-thio-GTP, and dGTP [85]. Interestingly, NUDT15 hydrolyzes 6-thio-dGTP and 6-thio-GTP more efficiently than it does GTP and dGTP. Moriyama et al. reported that NUDT15 converted the thiopurine active metabolites 6-thio-GTP and 6-thio-dGTP into 6-thio-GMP and 6-thiodGMP and that the variants of NUDT15 had lower enzyme activity [80]. This caused higher thiopurine active metabolite levels, resulting in thiopurine-induced 
leukopenia [80]. Therefore, the DNA-incorporated thioguanine (DNA-TG) metabolite was preferable to 6-TGN for adjusting thiopurine doses according to NUDT15 genotypes [86]. Valerie et al. reported that the enzyme efficiencies of the p.Arg139Cys variants were similar to that of the wild-type, but that p.Arg139Cys caused structural abnormalities and the mutant rapidly degraded in cells [87].

\section{Additional variants of NUDT15 and their enzymatic activities}

Moriyama et al. studied major haplotypes of NUDT15 and their enzyme activities by analyzing 270 children with acute lymphoblastic leukemia (ALL) in Guatemala, Singapore, and Japan [80]. These authors identified four coding variants in exons 1 and 3, including the previously reported p.Arg139Cys in exon 3, and defined six haplotype $(* 1$ to $* 6$ ) combinations of these four variants (Fig. 2). Three variants, Arg139His, Val18Ile, and p.Val18_Val19insGlyVal, reduce NUDT15 enzyme activity at similar levels (almost 75\%); no enzyme activity was observed in the p.Arg139Cys variant. Haplotype frequencies in the Korean cohort [88] and in Chinese patients with IBD [89], and estimated frequencies of the Japanese cohort study for the Tohoku Medical Megabank Project $[50,51,90]$ are summarized in Fig. 2. Individual haplotype and insertion/deletion data of the Japanese cohort were not available; therefore, the frequencies are based on singlenucleotide variant frequencies. Distributions of the haplotype frequencies were similar in these three populations, and the two variants in exon 1 are not rare in East Asian populations. The diplotype (combination of haplotypes that exist in each individual) is more important in clinical settings. Moriyama et al. classified patients into three diplotypic groups according to expected enzyme activities: normal $(* 1 / * 1)$, intermediate $(* 1 / * 2, * 1 / * 3, * 1 / * 4$, and $* 1 /$ $* 5)$, and low activity $(* 2 / * 3, * 3 / * 3$, and $* 3 / * 5)$ [80]. In their study, they showed differences in the tolerated 6-MP doses for each population. However, there were limited numbers of subjects in each population, and haplotype *6 and some of the estimated diplotypes were not observed. Moreover, the treatment goals and dosages of 6-MP for ALL treatments in children are very different from those required in patients with IBD; therefore it is difficult to

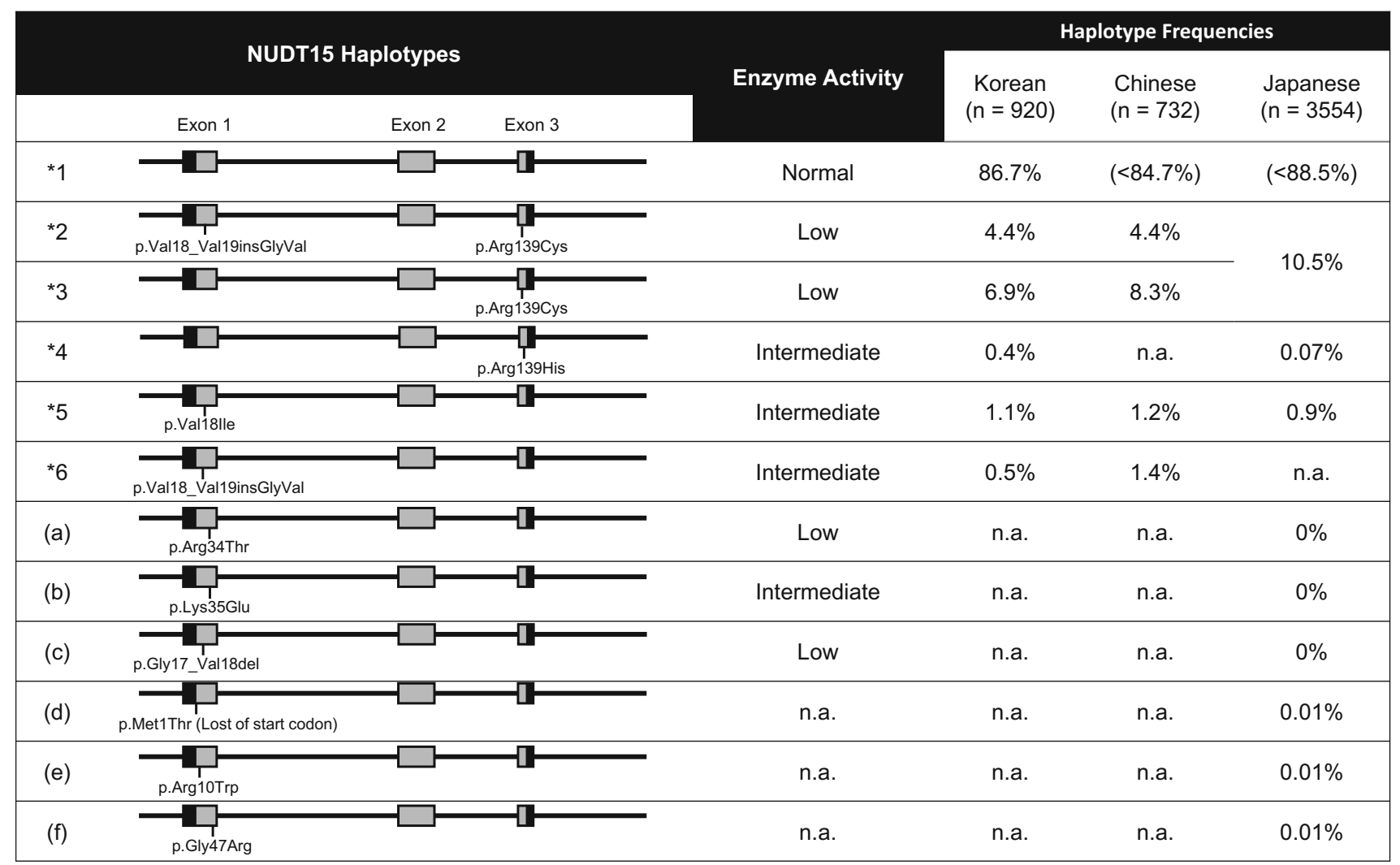

Fig. 2 Nucleoside diphosphate-linked moiety $X$-type motif 15 (NUDT15) haplotype structures and frequencies in East Asian populations. Common haplotypes $* 1$ to $* 6$ have been defined previously [81]. Rare variants (a), (b), (c) are from Moriyama et al.
[92], and rare variants (d), (e), and (f) and estimated haplotype frequencies in Japanese populations were from the $3.5 \mathrm{KJPN}$ data by the Tohoku Medical Megabank Project (https://ijgvd.megabank. tohoku.ac.jp/) [51, 52, 91]. n.a. data not available 
apply this classification directly to patients with IBD. Chao et al. recently reported associations between leukopenia and the NUDT15 diplotype in Chinese patients with IBD [89]. In this study, patients with $* 1 / * 6(n=20)$ or $* 2 / * 6$ diplotype $(n=1)$ were observed. The frequencies of leukopenia in these patients were 35.0 and $100 \%$, respectively, which is higher than the $15.1 \%$ in patients with the $* I / * I$ diplotype. The authors reported that the leukopenia frequencies appeared to be clearly related to NUDT15 enzyme activity based on diplotypes (Table 1).

More recently, an additional three variants, namely, p.Arg34Thr, p.Lys35Glu, and p.Gly17_Val18del, were observed in five children with ALL in Singapore, Taiwan, and the USA [91]. All of the children experienced reduced tolerance to 6-MP, and p.G17_V18del was only observed in children of European or African ancestries. This deletion is the first functional variant reported in European and African populations. Three other rare functional variants, i.e., p.Met1Thr (lost of start codon), p.Arg10Trp, and Gly47Arg, were observed in a Japanese cohort [50, 51], but their enzyme activities and association with thiopurineinduced adverse events are unknown. Many rare variants and additional haplotypes may exist, such as TPMT.

\section{Prospects for clinical application of NUDT15 genotyping}

All results to date indicate that thiopurine-induced leukopenia and severe alopecia are inevitable in patients homozygous for the p.Arg139Cys mutation. Consequently, p.Arg139Cys is a robust candidate for clinical applications aimed at predicting severe leukopenia and alopecia in patients with IBD. It remains unclear whether either the genotype of p.Arg139Cys or the diplotype of NUDT15 are preferable for clinical applications. If only p.Arg139Cys is tested, the activities of NUDT15 are expected to be normal (Arg/Arg), medium (Arg/Cys), or low (Cys/Cys) depending on the genotype. However, some diplotypes, such as $* 1 * 5, * 1 * 6, * 3 * 5$, and $* 2 * 6$, which have medium or low enzyme activities in vitro, cannot be correctly identified (Table 1). Chao et al. reported that the predictive sensitivity of NUDT15 p.Arg139Cys was $49.2 \%$ in their cohort of Chinese patients with IBD but that combined analysis with Val18Ile and p.Val18_Val19insGlyVal to determine diplotypes by detecting haplotypes $* 5$ and $* 6$ could increase the sensitivity to $55.4 \%$ [89]. This was the first study to examine the diplotype-based risk of thiopurines for IBD. However, the results should be interpreted with caution. Curiously, in the study of Chao et al. [89] the sensitivity of NUDT15 p.Arg139Cys for detecting leukopenia was lower than previously reported [72, 73, 82], possibly due to differences in leukopenia definitions in the various studies. In their study, Chao et al. [89] defined leukopenia as a white blood cell (WBC) count of $<3500 /$ $\mathrm{mm}^{3}$, which is relatively higher than levels reported elsewhere. Higher grade leukopenia (WBC $<2000 / \mathrm{mm}^{3}$ ) is clinically important because of severe associated infectious complications and high mortality rates associated with IBD [92]. It is unclear which grade of leukopenia the patients with each diplotype had, and it is also important to consider if they had alopecia given its severity, prolonged recovery times, and associated cosmetic issues.

For clinical applications, it is important to clearly decide on the target of the pharmacogenetic tests. Additionally, the time, effort, and costs of genotyping are important factors to take into consideration. Given the diplotype frequencies in Chinese patients with IBD and the estimated frequencies, based on haplotype frequencies, in the Korean and Japanese cohorts, undetectable diplotypes using the

Table 1 Nucleoside diphosphate-linked moiety $X$-type motif 15 diplotypes and leukopenia frequencies

\begin{tabular}{|c|c|c|c|c|c|c|c|c|c|c|c|c|}
\hline \multirow{2}{*}{$\frac{\text { NUDT15 enzyme activity [81] }}{\text { Diplotype }}$} & \multirow{2}{*}{$\frac{\text { Normal }}{* 1 * 1}$} & \multicolumn{5}{|c|}{ Medium } & \multicolumn{6}{|l|}{ Low } \\
\hline & & $* 1 * 4$ & $* 1 * 5$ & $* 1 * 6$ & $* 1 * 2$ & $* 1 * 3$ & $* 2 * 6$ & $* 2 * 5$ & $* 3 * 5$ & $* 2 * 2$ & $* 2 * 3$ & $* 3 * 3$ \\
\hline \multicolumn{13}{|l|}{ Diplotype frequencies } \\
\hline Chinese [90] (\%) & $<71.6$ & na & 1.78 & 2.73 & 7.8 & 13.9 & 0.14 & 0 & 0.55 & 0 & 0.82 & 0.68 \\
\hline Korean (estimated) ${ }^{\mathrm{a}}(\%)$ & 75.2 & 0.69 & 1.9 & 0.87 & 7.6 & 12.0 & 0.044 & 0.10 & 0.15 & 0.19 & 0.61 & 0.48 \\
\hline Japanese (estimated) $)^{\mathrm{b}}(\%)$ & $<78.3$ & 0.12 & 1.6 & n.a. & 18.6 & & n.a. & 0.19 & & 1.1 & & \\
\hline $\begin{array}{l}\text { Leukopenia frequencies [90] }(\%)(\mathrm{WBC}<3500 / \\
\mathrm{mm}^{3} \text {, Chinese) }\end{array}$ & 15.1 & n.a. & 30.8 & 35.0 & 49.1 & 42.2 & 100 & n.a. & 100 & n.a. & 100 & 100 \\
\hline p.Arg139Cys test result & $\begin{array}{l}\text { Normal } \\
\text { (Arg/ } \\
\text { Arg) }\end{array}$ & \multicolumn{3}{|c|}{$\begin{array}{l}\text { Normal } \\
\text { (Arg/Arg) }\end{array}$} & \multicolumn{2}{|c|}{$\begin{array}{l}\text { Medium } \\
\text { (Arg/Cys) }\end{array}$} & \multicolumn{3}{|c|}{$\begin{array}{l}\text { Medium } \\
\text { (Arg/Cys) }\end{array}$} & \multicolumn{3}{|c|}{$\begin{array}{l}\text { Low } \\
\text { (Cys/Cys) }\end{array}$} \\
\hline
\end{tabular}

NUDT15, Nucleoside diphosphate-linked moiety $X$-type motif 15; WBC, white blood cells; n.a., data not available

${ }^{a}$ Diplotype frequencies in Korean were estimated using haplotype frequencies reported by Kim et al. [89]

${ }^{\mathrm{b}}$ Diplotype frequencies in Japanese were estimated using single nucleotide polymorphism frequencies from $3.5 \mathrm{KJPN}$ data by the Tohoku Medical Megabank Project (https://ijgvd.megabank.tohoku.ac.jp/) [51, 52, 91] 
test for p.Arg139Cys are infrequent, and especially rare $(<1 \%)$ among low activity diplotypes $(* 2 * 6, * 2 * 5$, and $* 3 * 5)$. Therefore, we need to evaluate the genotyping-associated costs to identify these patients and examine their detailed clinical information, such as adverse event types and grades. If the target of the pharmacogenetic test is to screen for patients who will have severe leukopenia and alopecia, detecting p.Arg139Cys may suffice. If the target is to determine initial doses of thiopurines, detecting the diplotype of patients might be helpful. However, there is insufficient evidence, and further analyses with large cohorts and detailed clinical data are needed to confirm the clinical effects of these variants.

\section{Conclusion}

The mechanisms of thiopurine toxicity in Asian populations have been unclear for a long time, but were recently clarified by a valuable genome-wide association study in an Asian population. Given existing data pertaining to TPMT in a population of patients of European ancestry, testing the genotype or diplotype of NUDT15 is a good candidate in clinical applications for predicting thiopurine toxicity in Asian and Hispanic populations. However, further analyses will be needed to determine how and which variants should be genotyped, taking into account the allele frequencies in the target population. The costs and benefits of genotyping also require consideration. NUDT15 is a good model for showing that population-specific variants cause populationspecific drug intolerance. These results suggest that further population-specific pharmacogenetic studies are indicated.

Acknowledgements We would like to thank past and present members of the IBD group in Tohoku University for fruitful discussions and scientific contributions.

\section{Compliance with ethical standards}

Conflict of interest The authors declare that they have no conflict of interest.

Open Access This article is distributed under the terms of the Creative Commons Attribution 4.0 International License (http://crea tivecommons.org/licenses/by/4.0/), which permits unrestricted use, distribution, and reproduction in any medium, provided you give appropriate credit to the original author(s) and the source, provide a link to the Creative Commons license, and indicate if changes were made.

\section{References}

1. Jewell DP, Truelove SC. Azathioprine in ulcerative colitis: final report on controlled therapeutic trial. Br Med J. 1974;4:627-30.
2. Pearson DC, May GR, Fick GH, et al. Azathioprine and 6-mercaptopurine in Crohn disease. A meta-analysis. Ann Intern Med. 1995;123:132-42.

3. Hibi T, Naganuma M, Kitahora T, et al. Low-dose azathioprine is effective and safe for maintenance of remission in patients with ulcerative colitis. J Gastroenterol. 2003;38:740-6.

4. Gisbert JP, Nino P, Cara C, et al. Comparative effectiveness of azathioprine in Crohn's disease and ulcerative colitis: prospective, long-term, follow-up study of 394 patients. Aliment Pharmacol Ther. 2008;28:228-38.

5. Khan KJ, Dubinsky MC, Ford AC, et al. Efficacy of immunosuppressive therapy for inflammatory bowel disease: a systematic review and meta-analysis. Am J Gastroenterol. 2011;106:630-42.

6. Chande N, Patton PH, Tsoulis DJ, et al. Azathioprine or 6-mercaptopurine for maintenance of remission in Crohn's disease. Cochrane Database Syst Rev. 2015;10:CD000067. https://doi. org/10.1002/14651858.

7. Panaccione R, Ghosh S, Middleton S, et al. Combination therapy with infliximab and azathioprine is superior to monotherapy with either agent in ulcerative colitis. Gastroenterology. 2014;146:392-400.

8. Colombel JF, Sandborn WJ, Reinisch W, et al. Infliximab, azathioprine, or combination therapy for Crohn's disease. N Engl J Med. 2010;362:1383-95.

9. Qiu Y, Mao R, Chen BL, et al. Effects of combination therapy with immunomodulators on trough levels and antibodies against tumor necrosis factor antagonists in patients with inflammatory bowel disease: a meta-analysis. Clin Gastroenterol Hepatol. 2017;15:1359-72.

10. Jun JB, Cho DY, Kang C, et al. Thiopurine $S$-methyltransferase polymorphisms and the relationship between the mutant alleles and the adverse effects in systemic lupus erythematosus patients taking azathioprine. Clin Exp Rheumatol. 2005;23:873-6.

11. Qiu Y, Mao R, Zhang SH, et al. Safety profile of thiopurines in Crohn disease: analysis of 893 patient-years follow-up in a Southern China cohort. Medicine. 2015;94:e1513.

12. Takatsu N, Matsui T, Murakami Y, et al. Adverse reactions to azathioprine cannot be predicted by thiopurine $S$-methyltransferase genotype in Japanese patients with inflammatory bowel disease. J Gastroenterol Hepatol. 2009;24:1258-64.

13. Palmieri O, Latiano A, Bossa F, et al. Sequential evaluation of thiopurine methyltransferase, inosine triphosphate pyrophosphatase, and HPRT1 genes polymorphisms to explain thiopurines' toxicity and efficacy. Aliment Pharmacol Ther. 2007;26:737-45.

14. Gearry RB, Barclay ML, Burt MJ, et al. Thiopurine drug adverse effects in a population of New Zealand patients with inflammatory bowel disease. Pharmacoepidemiol Drug Saf. 2004; 13:563-7.

15. Ruemmele FM, Veres G, Kolho KL, et al. Consensus guidelines of ECCO/ESPGHAN on the medical management of pediatric Crohn's disease. J Crohns Colitis. 2014;8:1179-207.

16. Dubinsky MC, Lamothe S, Yang HY, et al. Pharmacogenomics and metabolite measurement for 6-mercaptopurine therapy in inflammatory bowel disease. Gastroenterology. 2000;118:705-13.

17. Haglund S, Taipalensuu J, Peterson C, et al. IMPDH activity in thiopurine-treated patients with inflammatory bowel disease-relation to TPMT activity and metabolite concentrations. Br J Clin Pharmacol. 2008;65:69-77.

18. Lennard L, Van Loon JA, Lilleyman JS, et al. Thiopurine pharmacogenetics in leukemia: correlation of erythrocyte thiopurine methyltransferase activity and 6-thioguanine nucleotide concentrations. Clin Pharmacol Ther. 1987;41:18-25.

19. Schutz E, Gummert J, Armstrong VW, et al. Azathioprine pharmacogenetics: the relationship between 6-thioguanine nucleotides 
and thiopurine methyltransferase in patients after heart and kidney transplantation. Eur $\mathrm{J}$ Clin Chem Clin Biochem. 1996;34:199-205.

20. Lorenz M, Weise A, Prause S, et al. Development and validation of a rapid and reliable method for TPMT genotyping using realtime PCR. Clin Lab. 2012;58:959-71.

21. Nasedkina TV, Fedorova OE, Glotov AS, et al. Rapid genotyping of common deficient thiopurine $S$-methyltransferase alleles using the DNA-microchip technique. Eur J Hum Genet. 2006;14:991-8.

22. Relling MV, Gardner EE, Sandborn WJ, et al. Clinical Pharmacogenetics Implementation Consortium guidelines for thiopurine methyltransferase genotype and thiopurine dosing. Clin Pharmacol Ther. 2011;89:387-91.

23. Derijks LJ, Gilissen LP, Engels LG, et al. Pharmacokinetics of 6-thioguanine in patients with inflammatory bowel disease. Ther Drug Monit. 2006;28:45-50.

24. Kurtovic S, Grehn L, Karlsson A, et al. Glutathione transferase activity with a novel substrate mimics the activation of the prodrug azathioprine. Anal Biochem. 2008;375:339-44.

25. Derijks LJ, Wong DR. Pharmacogenetics of thiopurines in inflammatory bowel disease. Curr Pharm Des. 2010;16:145-54.

26. Moon W, Loftus EV Jr. Review article: recent advances in pharmacogenetics and pharmacokinetics for safe and effective thiopurine therapy in inflammatory bowel disease. Aliment Pharmacol Ther. 2016;43:863-83.

27. Nelson JA, Carpenter JW, Rose LM, et al. Mechanisms of action of 6-thioguanine, 6-mercaptopurine, and 8-azaguanine. Cancer Res. 1975;35:2872-8.

28. Fairchild CR, Maybaum J, Kennedy KA. Concurrent unilateral chromatid damage and DNA strand breakage in response to 6-thioguanine treatment. Biochem Pharmacol. 1986;35:3533-41.

29. Poppe D, Tiede I, Fritz G, et al. Azathioprine suppresses ezrinradixin-moesin-dependent $\mathrm{T}$ cell-APC conjugation through inhibition of Vav guanosine exchange activity on Rac proteins. J Immunol. 2006;176:640-51.

30. Weinshilboum RM, Sladek SL. Mercaptopurine pharmacogenetics: monogenic inheritance of erythrocyte thiopurine methyltransferase activity. Am J Hum Genet. 1980;32:651-62.

31. Krynetski EY, Tai HL, Yates CR, et al. Genetic polymorphism of thiopurine $S$-methyltransferase: clinical importance and molecular mechanisms. Pharmacogenetics. 1996;6:279-90.

32. Colombel JF, Ferrari N, Debuysere H, et al. Genotypic analysis of thiopurine $S$-methyltransferase in patients with Crohn's disease and severe myelosuppression during azathioprine therapy. Gastroenterology. 2000;118:1025-30.

33. Al Hadithy AF, de Boer NK, Derijks LJ, et al. Thiopurines in inflammatory bowel disease: pharmacogenetics, therapeutic drug monitoring and clinical recommendations. Dig Liver Dis. 2005;37:282-97.

34. Seidman EG. Clinical use and practical application of TPMT enzyme and 6-mercaptopurine metabolite monitoring in IBD. Rev Gastroenterol Disord. 2003;3:S30-8.

35. Nygaard U, Toft N, Schmiegelow K. Methylated metabolites of 6-mercaptopurine are associated with hepatotoxicity. Clin Pharmacol Ther. 2004;75:274-81.

36. Kopylov U, Amre D, Theoret Y, et al. Thiopurine metabolite ratios for monitoring therapy in pediatric Crohn disease. J Pediatr Gastroenterol Nutr. 2014;59:511-5.

37. Ansari A, Hassan C, Duley J, et al. Thiopurine methyltransferase activity and the use of azathioprine in inflammatory bowel disease. Aliment Pharmacol Ther. 2002;16:1743-50.

38. de la Moureyre CS, Debuysere H, Sabbagh N, et al. Detection of known and new mutations in the thiopurine $S$-methyltransferase gene by single-strand conformation polymorphism analysis. Hum Mutat. 1998;12:177-85.
39. Iu YPH, Helander S, Kahlin AZ, et al. One amino acid makes a difference-characterization of a new TPMT allele and the influence of SAM on TPMT stability. Sci Rep. 2017;7:46428.

40. Ujiie S, Sasaki T, Mizugaki M, et al. Functional characterization of 23 allelic variants of thiopurine $S$-methyltransferase gene (TPMT*2-*24). Pharmacogenet Genomics. 2008;18:887-93.

41. Winter JW, Gaffney D, Shapiro D, et al. Assessment of thiopurine methyltransferase enzyme activity is superior to genotype in predicting myelosuppression following azathioprine therapy in patients with inflammatory bowel disease. Aliment Pharmacol Ther. 2007;25:1069-77.

42. Schaeffeler E, Eichelbaum M, Reinisch W, et al. Three novel thiopurine $S$-methyltransferase allelic variants (TPMT*20, *21, *22)-association with decreased enzyme function. Hum Mutat. 2006;27:976.

43. Lindqvist $\mathrm{M}$, Haglund $\mathrm{S}$, Almer $\mathrm{S}$, et al. Identification of two novel sequence variants affecting thiopurine methyltransferase enzyme activity. Pharmacogenetics. 2004;14:261-5.

44. Roberts RL, Gearry RB, Bland MV, et al. Trinucleotide repeat variants in the promoter of the thiopurine $S$-methyltransferase gene of patients exhibiting ultra-high enzyme activity. Pharmacogenet Genomics. 2008;18:434-8.

45. Yan L, Zhang S, Eiff B, et al. Thiopurine methyltransferase polymorphic tandem repeat: genotype-phenotype correlation analysis. Clin Pharmacol Ther. 2000;68:210-9.

46. Brouwer C, De Abreu RA, Keizer-Garritsen JJ, et al. Thiopurine methyltransferase in acute lymphoblastic leukaemia: biochemical and molecular biological aspects. Eur J Cancer. 2005;41:613-23.

47. Charasson V, Hillaire-Buys D, Solassol I, et al. Involvement of gene polymorphisms of the folate pathway enzymes in gene expression and anticancer drug sensitivity using the NCI-60 panel as a model. Eur J Cancer. 2009;45:2391-401.

48. Karas-Kuzelicki N, Milek M, Mlinaric-Rascan I. MTHFR and TYMS genotypes influence TPMT activity and its differential modulation in males and females. Clin Biochem. 2010;43:37-42.

49. Dorababu P, Naushad SM, Linga VG, et al. Genetic variants of thiopurine and folate metabolic pathways determine 6-MP-mediated hematological toxicity in childhood ALL. Pharmacogenomics. 2012;13:1001-8.

50. Nagasaki M, Yasuda J, Katsuoka F, et al. Rare variant discovery by deep whole-genome sequencing of 1,070 Japanese individuals. Nat Commun. 2015;6:8018.

51. Yamaguchi-Kabata Y, Nariai N, Kawai Y, et al. iJGVD: an integrative Japanese genome variation database based on wholegenome sequencing. Hum Genome Var. 2015;2:15050.

52. Uchiyama K, Nakamura M, Kubota $\mathrm{T}$, et al. Thiopurine $S$ methyltransferase and inosine triphosphate pyrophosphohydrolase genes in Japanese patients with inflammatory bowel disease in whom adverse drug reactions were induced by azathioprine/6mercaptopurine treatment. J Gastroenterol. 2009;44:197-203.

53. Kham SK, Soh CK, Aw DC, et al. TPMT*26 (208F->L), a novel mutation detected in a Chinese. $\mathrm{Br} \mathrm{J}$ Clin Pharmacol. 2009;68:120-3.

54. Sasaki T, Goto E, Konno Y, et al. Three novel single nucleotide polymorphisms of the human thiopurine $S$-methyltransferase gene in Japanese individuals. Drug Metab Pharmacokinet. 2006;21:332-6.

55. Ban H, Andoh A, Tanaka A, et al. Analysis of thiopurine $S$ methyltransferase genotypes in Japanese patients with inflammatory bowel disease. Intern Med. 2008;47:1645-8.

56. Wielinga PR, Reid G, Challa EE, et al. Thiopurine metabolism and identification of the thiopurine metabolites transported by MRP4 and MRP5 overexpressed in human embryonic kidney cells. Mol Pharmacol. 2002;62:1321-31. 
57. Krishnamurthy P, Schwab M, Takenaka K, et al. Transportermediated protection against thiopurine-induced hematopoietic toxicity. Cancer Res. 2008;68:4983-9.

58. Ban $\mathrm{H}$, Andoh $\mathrm{A}$, Imaeda $\mathrm{H}$, et al. The multidrug-resistance protein 4 polymorphism is a new factor accounting for thiopurine sensitivity in Japanese patients with inflammatory bowel disease. J Gastroenterol. 2010;45:1014-21.

59. Tanaka Y. Susceptibility to 6-mercaptopurine toxicity related with NUDT15 and ABCC4 variants in Japanese childhood acute lymphoblastic leukemia. Rinsho Ketsueki. 2017;58:950-6.

60. Tanaka Y, Nakadate $\mathrm{H}$, Kondoh $\mathrm{K}$, et al. Interaction between NUDT15 and ABCC4 variants enhances intolerability of 6-mercaptopurine in Japanese patients with childhood acute lymphoblastic leukemia. Pharmacogenomics J. 2017. https:/doi.org/ 10.1038/tpj.2017.12.

61. Holmes SL, Turner BM, Hirschhorn K. Human inosine triphosphatase: catalytic properties and population studies. Clin Chim Acta. 1979;97:143-53.

62. Lin S, McLennan AG, Ying K, et al. Cloning, expression, and characterization of a human inosine triphosphate pyrophosphatase encoded by the ITPA gene. J Biol Chem. 2001;276:18695-701.

63. Marinaki AM, Ansari A, Duley JA, et al. Adverse drug reactions to azathioprine therapy are associated with polymorphism in the gene encoding inosine triphosphate pyrophosphatase (ITPase). Pharmacogenetics. 2004;14:181-7.

64. Stocco G, Crews KR, Evans WE. Genetic polymorphism of inosine-triphosphate-pyrophosphatase influences mercaptopurine metabolism and toxicity during treatment of acute lymphoblastic leukemia individualized for thiopurine- $S$-methyl-transferase status. Expert Opin Drug Saf. 2010;9:23-37.

65. Gearry RB, Roberts RL, Barclay ML, et al. Lack of association between the ITPA 94C $>$ A polymorphism and adverse effects from azathioprine. Pharmacogenetics. 2004;14:779-81.

66. van Dieren JM, van Vuuren AJ, Kusters JG, et al. ITPA genotyping is not predictive for the development of side effects in AZA treated inflammatory bowel disease patients. Gut. 2005;54:1664.

67. Van Dieren JM, Hansen BE, Kuipers EJ, et al. Meta-analysis: Inosine triphosphate pyrophosphatase polymorphisms and thiopurine toxicity in the treatment of inflammatory bowel disease. Aliment Pharmacol Ther. 2007;26:643-52.

68. Kudo M, Saito Y, Sasaki T, et al. Genetic variations in the HGPRT, ITPA, IMPDH1, IMPDH2, and GMPS genes in Japanese individuals. Drug Metab Pharmacokinet. 2009;24:557-64.

69. Zhu Q, Cao Q. Thiopurine methyltransferase gene polymorphisms and activity in Chinese patients with inflammatory bowel disease treated with azathioprine. Chin Med J (Engl). 2012;125:3665-70.

70. Yang SK, Hong M, Baek J, et al. A common missense variant in NUDT15 confers susceptibility to thiopurine-induced leukopenia. Nat Genet. 2014;46:1017-20.

71. Tanaka Y, Kato M, Hasegawa D, et al. Susceptibility to 6-MP toxicity conferred by a NUDT15 variant in Japanese children with acute lymphoblastic leukaemia. $\mathrm{Br} \mathrm{J}$ Haematol. 2015;171:109-15.

72. Kakuta Y, Naito T, Onodera M, et al. NUDT15 R139C causes thiopurine-induced early severe hair loss and leukopenia in Japanese patients with IBD. Pharmacogenomics J. 2016;16:280-5.

73. Asada A, Nishida A, Shioya M, et al. NUDT15 R139C-related thiopurine leukocytopenia is mediated by 6-thioguanine nucleotide-independent mechanism in Japanese patients with inflammatory bowel disease. J Gastroenterol. 2016;51:22-9.

74. Zhu X, Wang XD, Chao K, et al. NUDT15 polymorphisms are better than thiopurine $S$-methyltransferase as predictor of risk for thiopurine-induced leukopenia in Chinese patients with Crohn's disease. Aliment Pharmacol Ther. 2016;44:967-75.

75. Ailing Z, Jing Y, Jingli L, et al. Further evidence that a variant of the gene NUDT15 may be an important predictor of azathioprineinduced toxicity in Chinese subjects: a case report. J Clin Pharm Ther. 2016;41:572-4.

76. Wong FC, Leung AW, Kwok JS, et al. NUDT15 variant and thiopurine-induced leukopenia in Hong Kong. Hong Kong Med J. 2016;22:185-7.

77. Liang DC, Yang CP, Liu HC, et al. NUDT15 gene polymorphism related to mercaptopurine intolerance in Taiwan Chinese children with acute lymphoblastic leukemia. Pharmacogenomics J. 2016;16:536-9.

78. Shah SA, Paradkar M, Desai D, et al. Nucleoside diphosphatelinked moiety X-type motif $15 \mathrm{C} 415 \mathrm{~T}$ variant as a predictor for thiopurine-induced toxicity in Indian patients. J Gastroenterol Hepatol. 2017;32:620-4.

79. Chiengthong K, Ittiwut C, Muensri S, et al. NUDT15 c. $415 \mathrm{C}>\mathrm{T}$ increases risk of 6-mercaptopurine induced myelosuppression during maintenance therapy in children with acute lymphoblastic leukemia. Haematologica. 2016;101:e24-6.

80. Moriyama T, Nishii R, Perez-Andreu V, et al. NUDT15 polymorphisms alter thiopurine metabolism and hematopoietic toxicity. Nat Genet. 2016;48:367-73.

81. Soler AM, Olano N, Mendez Y, et al. TPMT and NUDT15 genes are both related to mercaptopurine intolerance in acute lymphoblastic leukaemia patients from Uruguay. $\mathrm{Br} \mathrm{J}$ Haematol. 2017. https:/doi.org/10.1111/bjh.14532.

82. Sato T, Takagawa T, Kakuta Y, et al. NUDT15, FTO, and RUNX1 genetic variants and thiopurine intolerance among Japanese patients with inflammatory bowel diseases. Intest Res. 2017;15:328-37.

83. Yi ES, Choi YB, Choi R, et al. NUDT15 variants cause hematopoietic toxicity with low 6-TGN levels in children with acute lymphoblastic leukemia. Cancer Res Treat. 2017. doi: https://doi.org/10.4143/crt.2017.283.

84. Cai JP, Ishibashi T, Takagi Y, et al. Mouse MTH2 protein which prevents mutations caused by 8-oxoguanine nucleotides. Biochem Biophys Res Commun. 2003;305:1073-7.

85. Carter M, Jemth AS, Hagenkort A, et al. Crystal structure, biochemical and cellular activities demonstrate separate functions of MTH1 and MTH2. Nat Commun. 2015;6:7871.

86. Moriyama T, Nishii R, Lin TN, et al. The effects of inherited NUDT15 polymorphisms on thiopurine active metabolites in Japanese children with acute lymphoblastic leukemia. Pharmacogenet Genomics. 2017;27:236-9.

87. Valerie NC, Hagenkort A, Page BD, et al. NUDT15 hydrolyzes 6-Thio-DeoxyGTP to mediate the anticancer efficacy of 6-thioguanine. Cancer Res. 2016;76:5501-11.

88. Kim HT, Choi R, Won HH, et al. NUDT15 genotype distributions in the Korean population. Pharmacogenet Genomics. 2017;27:197-200.

89. Chao K, Wang X, Cao Q, et al. Combined detection of NUDT15 variants could highly predict thiopurine-induced leukopenia in Chinese patients with inflammatory bowel disease: a multicenter analysis. Inflamm Bowel Dis. 2017;23:1592-9.

90. Kuriyama S, Yaegashi N, Nagami F, et al. The Tohoku Medical Megabank Project: design and mission. J Epidemiol. 2016;26:493-511.

91. Moriyama T, Yang YL, Nishii R, et al. Novel variants in NUDT15 and thiopurine intolerance in children with acute lymphoblastic leukemia from diverse ancestry. Blood. 2017;130:1209-12.

92. Connell WR, Kamm MA, Ritchie JK, et al. Bone marrow toxicity caused by azathioprine in inflammatory bowel disease: 27 years of experience. Gut. 1993;34:1081-5. 